\title{
Late introduction of low dose resveratrol and grape powder after estradiol depletion does not restore glucose tolerance in the ovariectomized rat
}

\begin{abstract}
Eóin Anderson, Daniel T. Cervone, and David J. Dyck
Department of Human Health and Nutritional Sciences, University of Guelph, Guelph, Ontario, N1G2W1, Canada

Corresponding Author: Dr. David J. Dyck, PhD, Department of Human Health and Nutritional Sciences, University of Guelph, Guelph, Ontario, N1G2W1, Canada

Submission Date: November $8^{\text {th }}, 2017$, Acceptance Date: February $25^{\text {th }}$, 2018, Publication Date: February $28^{\text {th }}, 2018$

Citation: Anderson E., Cervone D.T., Dyck D.J., Late Introduction of Low Dose Resveratrol and Grape Powder After Estradiol Depletion Does Not Restore Glucose Tolerance in the Ovariectomized Rat, Functional Foods in Health and Disease 2018; 8(2):79-90. DOI: https://doi.org/10.31989/ffhd.v8i2.405
\end{abstract}

\begin{abstract}
Background: Estrogen (E2) loss is associated with insulin resistance. Natural compounds such as resveratrol (RESV) have potential insulin sensitizing effects. Grape pomace powder (GP) also contains RESV and other antioxidants. However, the ability of realistic, attainable concentrations of RESV and GP to reverse glucose intolerance in E2 deficient rats has not yet been explored.
\end{abstract}

Purpose: The aim of the current study was to determine whether RESV and GP, in realistic amounts that could be achieved with supplementation, would be effective in restoring glucose tolerance in the ovariectomized (OVX) rat. Furthermore, there appears to be a critical time window following the loss of E2 when hormonal replacement is effective, with delayed treatment being ineffective and potentially detrimental. Therefore, we were particularly interested in examining the effectiveness of RESV and GP as a delayed treatment (i.e. after the establishment of glucose intolerance) rather than administering at the onset of E2 loss.

Results: In the present study, rats demonstrated impaired glucose tolerance, as determined by an intraperitoneal glucose tolerance test, 12 weeks after bilateral ovary removal. Subsequently, OVX animals were randomly placed into a sham or one of 3 treatment groups. The treatments were

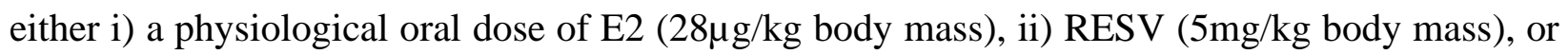
iii) GP (1.5g/100g of diet) for another 6 weeks. OVX animals were significantly heavier than nonOVX rats at the onset of glucose intolerance and this did not change throughout the treatment. None of the treatments restored glucose tolerance within the 6 weeks. Insulin tolerance did not 
worsen in OVX rats and was unaffected by treatment. Adipocyte size was generally increased in OVX animals and was not decreased with treatment.

Conclusion: In conclusion, delayed E2, RESV and GP treatment do not restore glucose tolerance in OVX rats. Low dose RESV and GP supplementation may not be effective alternatives to HRT to restore compromised glucose tolerance.

Keywords: ovariectomy, estrogen, resveratrol, grape pomace, glucose tolerance, insulin tolerance, delayed treatment

\section{BACKGROUND}

Premenopausal women are partially protected against insulin resistance and type 2 diabetes [1]. This protection is believed to be conferred by 17ß-estradiol (E2) [2]. E2 hormone replacement therapy has shown promise in rescuing metabolic function in animals when administered shortly after ovariectomy [3, 4]. However, there appears to be a critical time window following the loss of E2 when hormonal replacement is effective, after which treatment becomes ineffective and potentially detrimental [5-7]. E2 supplementation is also controversial due to the potential/perceived risk of cardiac events and breast cancer [8]. Therefore, alternative treatments are needed. We recently demonstrated that endurance treadmill exercise, initiated 10 weeks post ovariectomy, effectively restored glucose tolerance in female rats, while delayed E2 treatment only showed a trend towards improvement [9]. However, not all individuals are willing or able to exercise. Resveratrol (RESV) and grape pomace (GP) powder are commercially available supplements believed to have numerous potential health benefits, in part due to their polyphenol and antioxidant content.

RESV is a polyphenolic compound found in red grape skins [10]. The effects of RESV on glucose tolerance in humans have been mixed, with some studies showing no effect [11-13] and others demonstrating improved insulin sensitivity $[14,15]$. Rodent studies have depicted more clear benefits, including improved skeletal muscle glucose uptake [16] and hepatic insulin signaling [17]. Interestingly, any potential beneficial effect of RESV does not appear to be related to the dosage used. In obese humans, $10 \mathrm{mg} /$ day has been reported to improve insulin response [14] but $500 \mathrm{mg} /$ day has not [11]. In rats, dosages of $2.5-10 \mathrm{mg} / \mathrm{kg}$ administered for $4-8$ weeks, similar to that used in our current study, have been shown to improve insulin response $[17,18]$. To our knowledge, the efficacy of realistically attainable RESV concentrations (i.e. readily achieved through commercially available supplements) in restoring glucose tolerance in an E2deficient animal has not been explored.

GP is a byproduct of wine making and is composed of grape skins, seeds, and stems, in addition to containing RESV [10]. Grape pomace has been shown to be an antioxidant (Zhu et al. 2015) and also contains anthocyanin and quercetin, which have anti-inflammatory properties [19, 20]. GP, supplemented at $1 \mathrm{~g} / \mathrm{kg}$ body mass (similar to the dosage used in the current study) has been shown to protect fructose fed rats from insulin resistance [21]. To our knowledge the efficacy of GP in restoring glucose tolerance in OVX animals has not been explored.

The aim of the current study was to determine whether RESV and GP, in realistic amounts that could be achieved with supplementation (and also previously shown to be effective), would restore normal glucose tolerance in the ovariectomized rat. Given that RESV is commonly 
available in $500 \mathrm{mg}$ capsules, a normalized dose of approximately $5 \mathrm{mg} / \mathrm{kg}$ was considered a realistic amount. Furthermore, given the apparent critical window of treating E2 loss, we were interested in examining their effectiveness as a delayed treatment, rather than administering at the onset of E2 loss.

\section{METHODS}

\section{Materials and reagents}

17ß-Estradiol Molecular Assay Kit (Cat. \#ab108667) was purchased from Abcam (Toronto, ON, Canada). Resveratrol was purchased from Cayan Chemical Company (Michigan, USA) and Bioflavia grape pomace powder was kindly supplied by Southbrook Vineyards (Ontario, Canada).

\section{Animals}

All procedures were approved by the Animal Care Committee at the University of Guelph and followed Canadian Council of Animal Care guidelines. Female Sprague Dawley rats were purchased from Charles River Laboratories at 2 months of age (body mass, $208 \pm 1 \mathrm{~g}$ ). Two days prior to arrival, all rats underwent either bilateral ovariectomy (OVX; n=40) or SHAM surgery (SHAM; $\mathrm{n}=10$ ) by Charles River technicians. OVX surgeries were verified following terminal experiments by the assessment of plasma E2 content.

Two to three animals were grouped in cages of either SHAM or OVX for the first 12 weeks, in a temperature controlled room $\left(25^{\circ} \mathrm{C}\right)$ with a $12: 12$-h standard light-dark cycle and ad libitum access to food and water. All animals were fed a phytoestrogen-purified, soy protein-free diet (Harlan 2020X) which provided $60 \%$ of calories from carbohydrate, $24 \%$ from protein, and $16 \%$ from fat. Food intake and body mass were recorded weekly throughout the study.

\section{Initial Glucose Tolerance Tests}

At the end of the initial 12 weeks, the establishment of glucose intolerance in the OVX rats was confirmed by an intraperitoneal glucose tolerance test (IPGTT) after an 8 hour fast. A bolus of glucose $(2.0 \mathrm{~g} / \mathrm{kg}$ body mass) was injected into the intraperitoneal cavity of the animal and blood glucose was measured from the tail vein at $0,15,30,45,60,90$, and 120 minutes using a handheld glucometer (FreeStyle Lite). The incremental area under the curve (AUC) for the duration for the glucose response was calculated.

\section{Treatment Period}

At the end of 12 weeks, at the point when glucose intolerance had been established, OVX animals were randomly assigned to one of a non-treatment (OVX-CON), or 3 treatment groups (OVX-E2, OVX-RESV, and OVX-GP). A non-OVX SHAM group was included for comparison. Ten animals were assigned to each group and it was confirmed that the average glucose AUC of each OVX group was statistically similar. The OVX-E2 group received a daily physiological dose of powdered E2 $(28 \mu \mathrm{g} / \mathrm{kg}$ body mass) mixed into a Nutella cream solution $(5 \mu \mathrm{L}$ sesame oil/1g Nutella/kg body mass) as previously described [22]. The OVX-RESV group received a daily dose of powdered RESV (5mg/kg body mass) mixed into the Nutella cream solution. Finally, the OVXGP group received powdered GP at $1.5 \mathrm{~g} / 100 \mathrm{~g}$ of diet, similar to other studies $[23,24]$. The 
composition of the powdered GP is shown in Table 1, and provides approximately $0.02 \mathrm{mg} / \mathrm{kg}$ body mass / day of RESV.

\section{Final GTT and Insulin Tolerance Test}

After 6 weeks, animals were administered another IPGTT (2.0 g glucose/kg body mass). Blood glucose was measured at the same time points as the initial test. Two days later, an intraperitoneal insulin tolerance test was performed $(0.5 \mathrm{U} / \mathrm{kg}$ body mass $)$ approximately 3 hours after the removal of food. Blood glucose was measured at 15 minute intervals for 1 hour. Area above the curve was determined for each animal and used to assess insulin tolerance between groups.

Table 1. Grape Powder/Pomace Composition

\begin{tabular}{|l|l|}
\hline \multicolumn{2}{|l|}{ Grape Pomace Composition As \% of Whole } \\
\hline Vitis Vinifera Grape Skin Powder & $90 \%$ \\
\hline Vitis Vinifera Grape Powder & $7 \%$ \\
\hline Vitamin C (Ascorbic Acid) & $3 \%$ \\
\hline Fiber & $40 \%$ \\
\hline Bioactive Components & $2.1 \%$ \\
\hline Anthocyanins & $0.013 \%$ \\
\hline Quercetins & $0.003 \%$ \\
\hline Resveratrol & \\
\hline
\end{tabular}

Supplemented into diet at $1.5 \%$ provides a dose of about $0.58 \mathrm{~g} / \mathrm{kg} / \mathrm{day}$.

\section{Terminal Surgeries}

Two to three days after the completion of the tolerance tests, terminal surgeries were performed. Visceral (retroperitoneal) adipose tissue was collected and immediately frozen in liquid nitrogen and stored at $-80^{\circ} \mathrm{C}$. Animals were anesthetized prior to all surgical procedures with an intraperitoneal injection of sodium pentobarbital ( $60 \mathrm{mg} / \mathrm{kg}$ body mass). After surgery, the animals were sacrificed with an intracardiac injection of pentobarbital.

\section{Adipocyte Size}

The average adipocyte size was calculated using AdipoSoft software v. 1.13 (University of Navarra, Pamplona, Spain). Adipose tissue samples from each animal ( $n=8$ from each of the 5 groups) were stained with modified Harris hematoxylin and eosin with phloxine. Images were captured using an Olympus FSX 100 light microscope, Olympus, Tokyo, Japan). Images containing approximately 300 cells were used to assess the cross sectional diameter of the cells.

\section{Estradiol Concentration}

Serum E2 concentrations were determined using an estradiol specific enzyme-linked immunosorbent assay (ELISA) kit according to manufacturer's instructions. 


\section{Statistics}

All statistics were analyzed using GraphPad Prism 6. A one-way analysis of variance (ANOVA) was used to compare groups. In the instances of repeated time points, or pre and post-insulin treatment, a repeated measures ANOVA was used. A Tukey's posthoc test was used to determine significant differences between groups if there was a significant main effect or interaction revealed by the ANOVA. In all tables and figures, letters are used to denote statistical significance such that groups sharing a letter are not significantly different from one another. Significance was accepted at $\mathrm{p} \leq 0.05$. The data is presented as a mean \pm standard error (SE).

\section{RESULTS}

\section{Ovariectomy results in hyperphagia and increased body mass}

Ovariectomy resulted in significant hyperphagia from the start of the initial 12 weeks (i.e. induction of glucose intolerance period) ( $<<0.001$; Table 2), but tapered off after 5 weeks. The body mass of animals in all OVX groups was significantly higher than SHAMs by the second week of the induction period ( $\mathrm{p}<0.0001$; Table 1), which continued until the end of the treatment period. None of the treatments resulted in significant changes in body mass in the OVX rats.

Table 2. Body Mass and Food Intake

\begin{tabular}{|c|c|c|c|c|c|}
\hline Treatment Group & SHAM & OVX-CON & OVX-E2 & OVX-RESV & OVX-GP \\
\hline Initial Mass (g) & $235.4 \pm 4.2$ & $268.5 \pm 3.8$ & $269.9 \pm 3.8$ & $266.3 \pm 4.5$ & $266.4 \pm 4.4$ \\
\hline Mass at Treatment $(\mathrm{g})$ & $349.6 \pm 12.4$ & $422.9 \pm 16.0^{b}$ & $428.9 \pm 14.9^{b}$ & $437.1 \pm 16.3^{b}$ & $429.4 \pm 16.5^{b}$ \\
\hline Final Mass (g) & $364.9 \pm 12.9^{\mathrm{a}}$ & $432.8 \pm 16.8^{b}$ & $437.1 \pm 18.5^{b}$ & $451.2 \pm 18.7^{b}$ & $429.6 \pm 14.6^{\mathrm{b}}$ \\
\hline $\begin{array}{l}\text { Mean Food Intake } \\
\text { Prior to Treatment } \\
\text { (g/wk) }\end{array}$ & $129.1 \pm 2.4$ & $141.3 \pm 4.4$ & $141.9 \pm 4.1$ & $147.6 \pm 4.1$ & $146.1 \pm 4.2$ \\
\hline $\begin{array}{l}\text { Mean Food Intake } \\
\text { During Treatment } \\
\text { (g/wk) }\end{array}$ & $127.0 \pm 4.6$ & $111.2 \pm 4.8$ & $109.6 \pm 4.8$ & $120.7 \pm 4.4$ & $113.3 \pm 3.4$ \\
\hline
\end{tabular}

Data are presented as mean \pm standard error, $n=10$ for all groups. Groups which share a letter are not statistically different. Statistical significance was accepted at $\mathrm{p}<0.05$. 
Plasma E2 concentration is significantly reduced following OVX but is restored with E2 supplementation

Plasma E2 was reduced by approximately 4-fold ( $\mathrm{p}=0.02)$ in OVX animals (Figure 1). E2 supplementation increased plasma E2 concentrations to be similar to the SHAM rats.

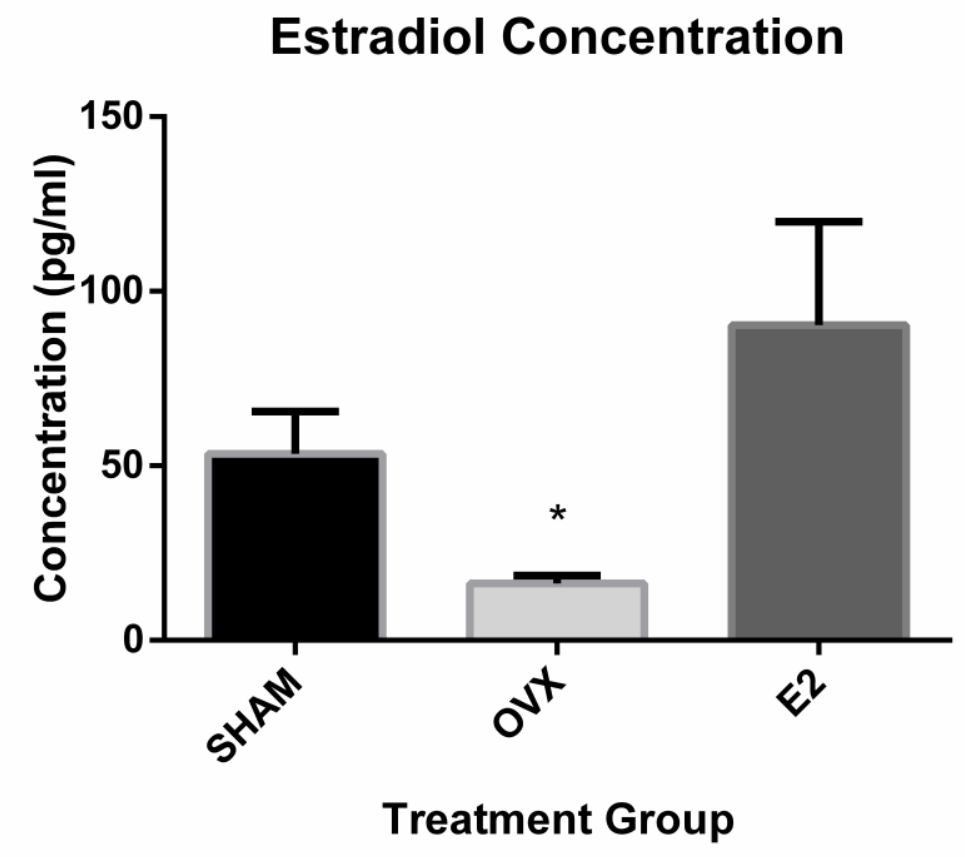

Figure 1. Plasma estrogen content. E2 plasma content is expressed in $\mathrm{pg} / \mathrm{mL}$ and data are presented as mean \pm standard error. Sample sizes are SHAM $(n=8)$, OVX $(n=23)$, and OVX E2 $(n=7)$. All OVX rats were combined for this assessment. Statistical significance was accepted at $\mathrm{p}<0.05$.

Glucose tolerance is impaired with E2 loss, but not restored by delayed E2 or nutritional intervention

The incremental glucose AUC was significantly increased $(\mathrm{p}=0.03)$ in OVX animals compared to SHAMs by 12 weeks post-ovariectomy, demonstrating a worsening of glucose tolerance (Figure 2). This impaired glucose tolerance remained after 6 weeks of treatment, regardless of treatment type (E2, RESV, or GP).

\section{Insulin tolerance is not different between groups}

To determine whether the impaired glucose tolerance in the OVX animals might be due to a loss of insulin action, an intraperitoneal ITT was conducted. Insulin tolerance, as determined by the calculated glucose area above the curve, was not significantly different between SHAM and any of the OVX groups (Figure 3). Therefore, changes in insulin tolerance cannot explain the worsening of glucose tolerance observed in OVX versus SHAM animals. 

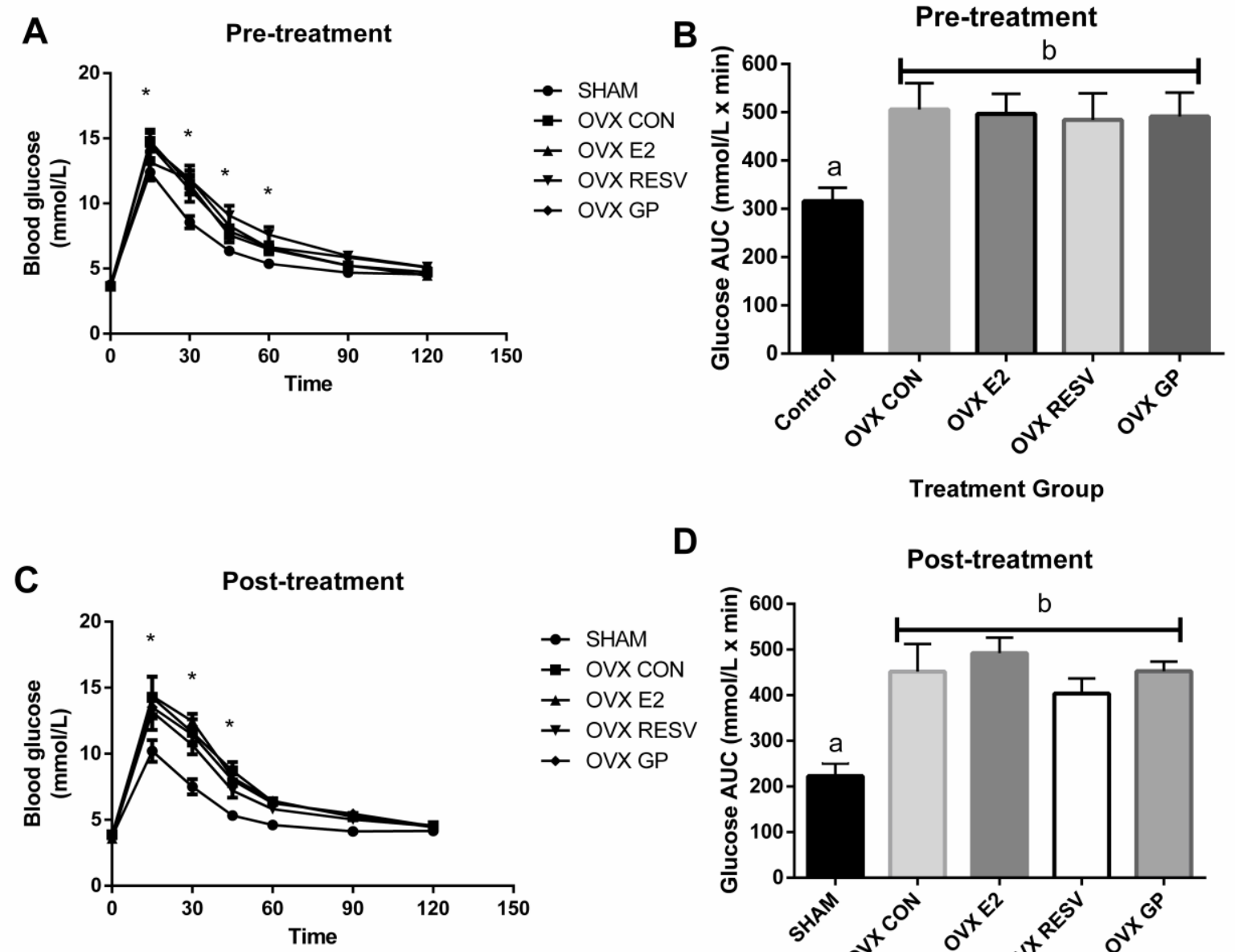

D
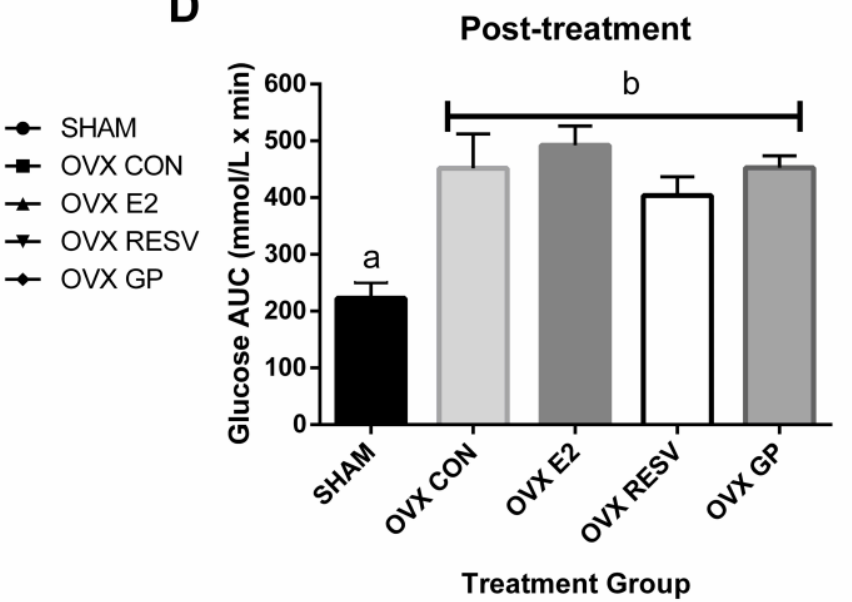

Figure 2. Glucose tolerance test ( $\mathrm{A}$ and $\mathrm{C}$ ) and calculated area under the curve (B and D) prior to, and after treatment in OVX animals. Data are presented as mean \pm standard error, $\mathrm{n}=10$ for all groups. Groups which share a letter are not statistically different. Statistical significance was accepted at $\mathrm{p}<0.05$.
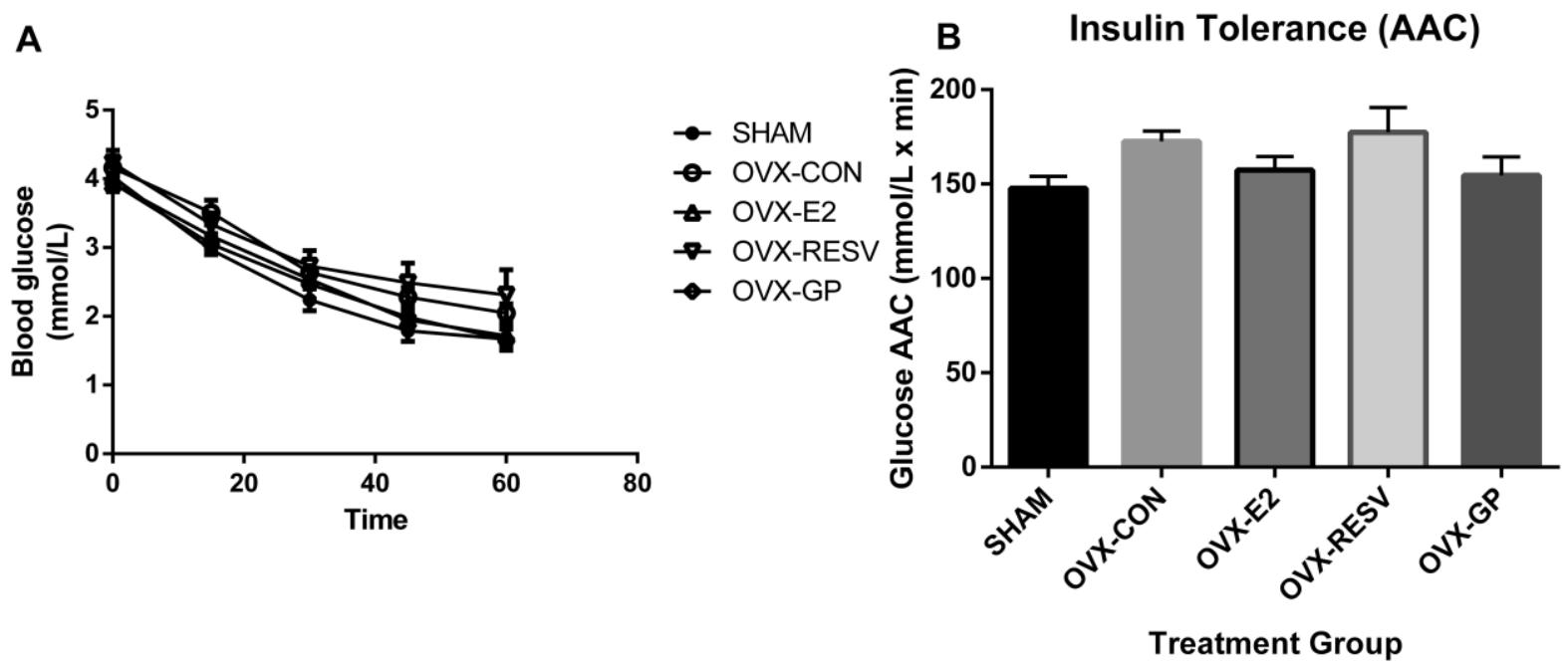

Figure 3. Insulin tolerance test $(A)$ and calculated area above the curve $(B)$ in OVX-treated animals. Data are presented as mean \pm standard error, $n=10$ for all groups. No groups were significantly different at $\mathrm{p}<0.05$. 
Adipocyte size is generally larger in OVX groups

OVX animals had 23 to $46 \%$ larger adipocytes than SHAM animals (Figure 4), although this was statistically significant only in OVX-E2 ( $\mathrm{p}=0.055)$ and OVX-RESV groups $(\mathrm{p}=0.012)$.
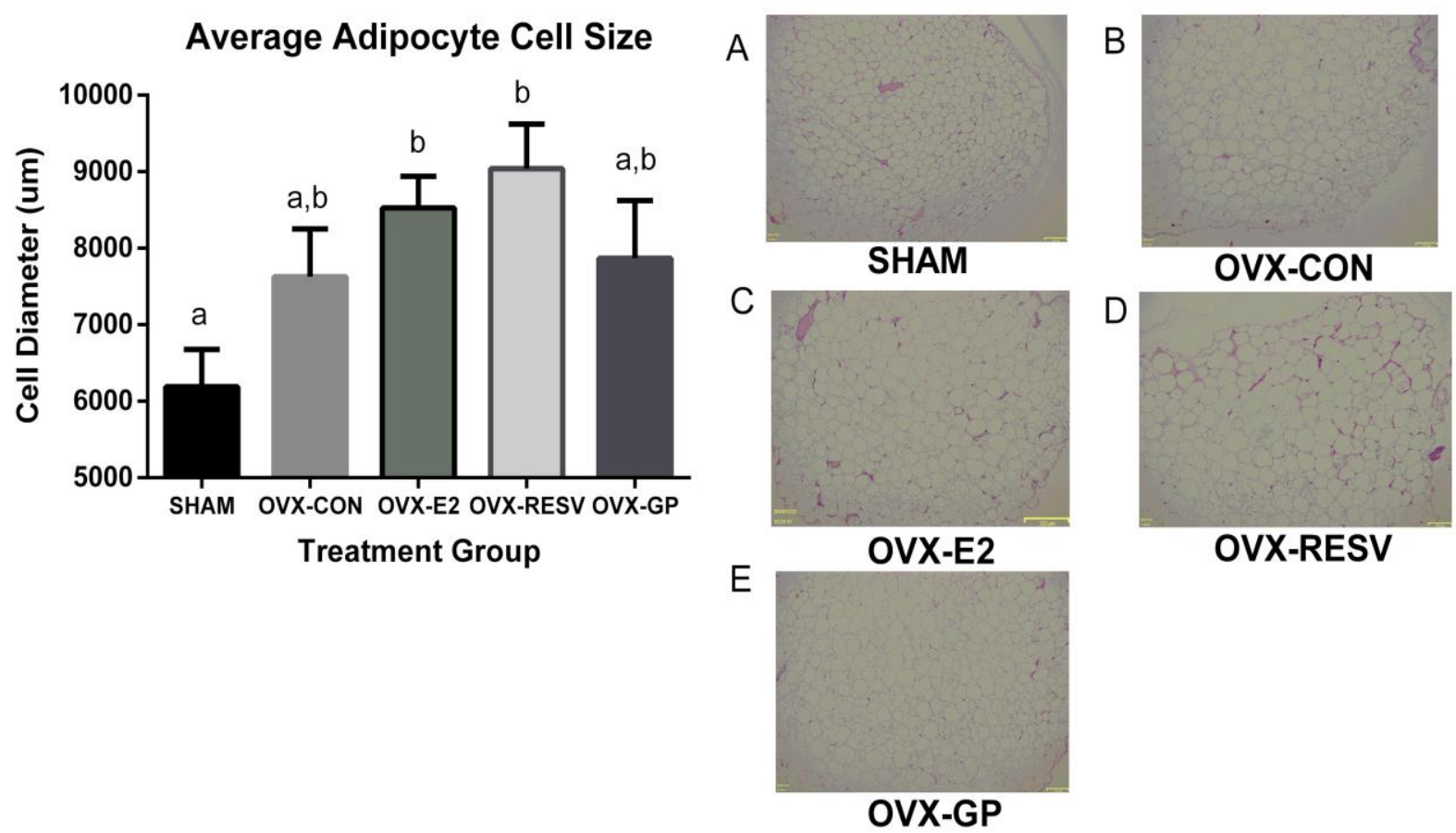

Figure 4. Visceral adipocyte diameter in OVX-treated animals. Cell size is expressed as the average diameter of a cell in $\mu \mathrm{m}$. Data are presented as mean \pm standard error, $n=8$ for all groups. Groups which share a letter are not statistically different. Statistical significance was accepted at $\mathrm{p}<0.05$. Representative images are A) SHAM, B) OVX CON, C) OVX-E2

\section{DISCUSSION}

The effectiveness of E2 replacement varies depending on how early repletion begins [6]. Our findings demonstrate that 12 weeks after ovariectomy, a further six weeks of oral E2, RESV, or GP treatment does not improve the impaired glucose tolerance in ovariectomized animals. The observed impairment in glucose tolerance does not appear to be due to reduced to the whole body insulin action. Our findings suggest that low dose RESV and GP may not represent effective nutraceuticals for improving the impaired glucose tolerance already established in physiological states of reduced E2. However, we acknowledge that a longer duration of supplementation, or higher doses, may confer benefits not observed within the 6 weeks used in the current study.

Previous research from our laboratory using the same E2 dose and method of delivery showed a slight (20\%), but non-significant improvement in glucose tolerance in OVX animals when E2 treatment was delayed 10 weeks after ovariectomy [9]. The animals in the present study had a relatively longer period of E2 depletion (12 weeks) before treatment began, compared to successful studies which began E2 repletion only 1-3 weeks post ovariectomy [25, 26]. This suggests longer periods of E2 deficiency lead to altered metabolism which becomes more difficult to correct. Other animal studies which have looked at rat hippocampal responsiveness to E2 [27] or cardiovascular disease risk [28] have found that E2 repletion is only effective if initiated shortly after E2 depletion. 
The results of studies in which RESV is supplemented to glucose intolerant rodents and humans have been mixed. Interestingly, demonstrating a beneficial effect on glucose tolerance does not appear to be related to the dosage given (i.e. range of $2.5 \mathrm{mg} / \mathrm{kg}$ body mass to $4 \mathrm{~g} / \mathrm{kg}$ body mass) $[16,17,29]$. In the present study, we used a relatively low dosing regimen of $5 \mathrm{mg} / \mathrm{kg}$ body mass in order to represent an attainable dose in humans through commercially available supplements. This is the first study that has investigated the effects of RESV on whole body glucose tolerance in an OVX animal model. One human study which investigated the effects of RESV supplementation in healthy, postmenopausal women also found no effect of treatment [13]. To our knowledge, this is also the first study to investigate GP's ability to rescue glucose tolerance in an E2-deficient model. As with RESV, it may be possible that GP is more effective when administered simultaneously to or shortly after the induction of the ovariectomy as a means of prevention, although this remains to be determined. Another factor that is important to consider is that the RES and antioxidant content varies significantly between different GP manufacturers and grape species $[10,30]$. The GP used in this study was a commercially available product from a nearby vineyard and had a lower amount of RESV compared to that used in other animal studies $(30 \mu \mathrm{g} / \mathrm{g}$ vs. $60 \mu \mathrm{g} / \mathrm{g}[23,24])$, but contains a significant concentration of anthocyanins and quercetins.

Food intake was significantly greater immediately following ovariectomy, but this effect was lost after 6 weeks, similar to that previously observed in rats [31]. In spite of the fact that food intake eventually was similar in OVX and SHAM groups, the increase in body mass of OVX animals only became significant by the $3^{\text {rd }}$ week post-surgery and remained greater throughout the treatment period, suggesting that there was a change in metabolic efficiency. Visceral adipose was analyzed as OVX animals tend to have increases in the content of visceral fat, which is more metabolically active and detrimental to whole body insulin action than subcutaneous fat [32]. Visceral adipocyte size was generally increased in OVX animals, although this was only statistically significant in OVX-E2 and OVX-RESV groups (38\% and 46\% larger respectively). It has been found in previous studies that oral E2 administration results in increased fat mass [33] which is in agreement with our observations in the OVX-E2 group.

\section{CONCLUSION}

In conclusion, this study is the first to investigate the effects of delayed administration of realistically attainable dosages of RESV and GP on glucose tolerance in an E2-deficient model. Delayed treatment for 6 weeks with low dose RESV and GP were not effective at rescuing the impaired glucose tolerance caused by E2 deficiency in female rats. Therefore, low dose RESV and GP supplementation may not be effective treatments to improve the glucose intolerance already established from prolonged E2 deficiency. However, we acknowledge the possibility that either higher dosages or low dosages administered for a longer period of time may have been effective. These possibilities should be tested.

List of Abbreviations: AAC, area above the curve; ANOVA, analysis of variance; AUC, area under the curve; E2, estradiol; ELISA, enzyme-linked immunosorbent assay; GP, grape pomace; 
IPGTT, intraperitoneal glucose tolerance test; IPITT, intraperitoneal insulin tolerance test; OVX, ovariectomy; RESV, resveratrol.

Authors' Contributions: EA and DD each contributed to the design of the study. EA and DC conducted the study. EA performed all of the analyses. EA and DD prepared the manuscript and all authors contributed to proofreading and revisions.

Competing Interests: There are no conflicts of interest to declare.

Acknowledgements and Funding: This study was funded by the Ontario Ministry of Agriculture, Food and Rural Affairs. Grape pomace powder was generously supplied by Southbrook Vineyards, Niagara-on-the-Lake, Ontario. Daniel Cervone was supported by a scholarship from the Natural Sciences and Engineering Research Council of Canada.

\section{REFERENCES}

1. Mauvais-Jarvis F, Clegg DJ, Hevener AL: The role of estrogens in control of energy balance and glucose homeostasis. Endocr Rev 2013, 34: 309-338.

2. Foryst-Ludwig A, Kintscher U: Metabolic impact of estrogen signalling through ERalpha and ERbeta. J Steroid Biochem Mol Biol 2010, 122: 74-81.

3. Cavalcanti-de-Albuquerque JP, Salvador IC, Martins EL, Jardim-Messeder D, Werneck-deCastro JP, Galina A, Carvalho DP: Role of estrogen on skeletal muscle mitochondrial function in ovariectomized rats: a time course study in different fiber types. J Appl Physiol (1985) 2014, 116:779-789.

4. Zhu L, Brown WC, Cai Q, Krust A, Chambon P, McGuinness OP, Stafford JM: Estrogen treatment after ovariectomy protects against fatty liver and may improve pathway-selective insulin resistance. Diabetes 2013, 62: 424-434.

5. Mangan G, Iqbal S, Hubbard A, Hamilton V, Bombardier E, Tiidus PM: Delay in postovariectomy estrogen replacement negates estrogen-induced augmentation of post-exercise muscle satellite cell proliferation. Can J Physiol Pharmacol 2015, 93: 945-951.

6. Pereira RI, Casey BA, Swibas TA, Erickson CB, Wolfe P, Van Pelt RE: Timing of Estradiol Treatment After Menopause May Determine Benefit or Harm to Insulin Action. J Clin Endocrinol Metab 2015, 100: 4456-4462.

7. Rocca WA, Grossardt BR, Shuster LT: Oophorectomy, menopause, estrogen treatment, and cognitive aging: clinical evidence for a window of opportunity. Brain Res 2011, 1379: 188198.

8. Rossouw JE, Anderson GL, Prentice RL, LaCroix AZ, Kooperberg C, Stefanick ML, Jackson RD, et al.: Writing Group for the Women's Health Initiative I: Risks and benefits of estrogen plus progestin in healthy postmenopausal women: principal results From the Women's Health Initiative randomized controlled trial. JAMA 2002, 288: 321-333.

9. MacDonald TL, Ritchie KL, Davies S, Hamilton MJ, Cervone DT, Dyck DJ: Exercise training is an effective alternative to estrogen supplementation for improving glucose homeostasis in ovariectomized rats. Physiol Rep 2015, 3. 
10. Yang J, Martinson TE, Liu RH: Phytochemical profiles and antioxidant activities of wine grapes. Food Chemistry 2009, 116: 332-339.

11. Poulsen MM, Vestergaard PF, Clasen BF, Radko Y, Christensen LP, Stodkilde-Jorgensen $\mathrm{H}$, Moller N, et al.: High-dose resveratrol supplementation in obese men: an investigatorinitiated, randomized, placebo-controlled clinical trial of substrate metabolism, insulin sensitivity, and body composition. Diabetes 2013, 62: 1186-1195.

12. Timmers S, De Ligt M, Phielix E, Van de Weijer T, Hansen J, Moonen-Kornips E, Schaart G, et al.: Resveratrol as Add-on Therapy in Subjects With Well-Controlled Type 2 Diabetes: A Randomized Controlled Trial. Diabetes Care 2016, 39: 2211-2217.

13. Yoshino J, Conte C, Fontana L, Mittendorfer B, Imai S, Schechtman KB, Gu C, et al.: Resveratrol supplementation does not improve metabolic function in nonobese women with normal glucose tolerance. Cell Metab 2012, 16: 658-664.

14. Brasnyo P, Molnar GA, Mohas M, Marko L, Laczy B, Cseh J, Mikolas E, et al.: Resveratrol improves insulin sensitivity, reduces oxidative stress and activates the Akt pathway in type 2 diabetic patients. Br J Nutr 2011, 106: 383-389.

15. Timmers S, Konings E, Bilet L, Houtkooper RH, Van de Weijer T, Goossens GH, Hoeks J, et al.: Calorie restriction-like effects of 30 days of resveratrol supplementation on energy metabolism and metabolic profile in obese humans. Cell Metab 2011, 14: 612-622.

16. Andersen G, Burkon A, Sulzmaier FJ, Walker JM, Leckband G, Fuhst R, Erbersdobler HF, et al.: High dose of dietary resveratrol enhances insulin sensitivity in healthy rats but does not lead to metabolite concentrations effective for SIRT1 expression. Mol Nutr Food Res 2011, 55: 1197-1206.

17. Gonzalez-Rodriguez A, Santamaria B, Mas-Gutierrez JA, Rada P, Fernandez-Millan E, Pardo V, Alvarez C, et al.: Resveratrol treatment restores peripheral insulin sensitivity in diabetic mice in a sirt1-independent manner. Mol Nutr Food Res 2015, 59: 1431-1442.

18. Yonamine CY, Pinheiro-Machado E, Michalani ML, Freitas HS, Okamoto MM, CorreaGiannella ML, Machado UF: Resveratrol improves glycemic control in insulin-treated diabetic rats: participation of the hepatic territory. Nutr Metab (Lond) 2016, 13: 44.

19. Chuang CC, Martinez K, Xie G, Kennedy A, Bumrungpert A, Overman A, et al.: Quercetin is equally or more effective than resveratrol in attenuating tumor necrosis factor- $\{$ alpha $\}$ mediated inflammation and insulin resistance in primary human adipocytes. Am J Clin Nutr 2010, 92: 1511-1521.

20. Li D, Zhang Y, Liu Y, Sun R, Xia M: Purified anthocyanin supplementation reduces dyslipidemia, enhances antioxidant capacity, and prevents insulin resistance in diabetic patients. J Nutr 2015, 145: 742-748.

21. Rodriguez Lanzi C, Perdicaro DJ, Antoniolli A, Fontana AR, Miatello RM, Bottini R, Vazquez Prieto MA: Grape pomace and grape pomace extract improve insulin signaling in high-fat-fructose fed rat-induced metabolic syndrome. Food Funct 2016, 7: 1544-1553.

22. Strom JO, Theodorsson A, Ingberg E, Isaksson IM, Theodorsson E: Ovariectomy and 17beta-estradiol replacement in rats and mice: a visual demonstration. J Vis Exp 2012, e4013. 
23. Hogan S, Canning C, Sun S, Sun X, Zhou K: Effects of grape pomace antioxidant extract on oxidative stress and inflammation in diet induced obese mice. J Agric Food Chem 2010, 58: 11250-11256.

24. Hogan S, Zhang L, Li J, Sun S, Canning C, Zhou K: Antioxidant rich grape pomace extract suppresses postprandial hyperglycemia in diabetic mice by specifically inhibiting alphaglucosidase. Nutr Metab (Lond) 2010, 7: 71.

25. Alonso A, Gonzalez-Pardo H, Garrido P, Conejo NM, Llaneza P, Diaz F, et al.: Acute effects of 17 beta-estradiol and genistein on insulin sensitivity and spatial memory in aged ovariectomized female rats. Age (Dordr) 2010, 32: 421-434.

26. Moreno M, Ordonez P, Alonso A, Diaz F, Tolivia J, Gonzalez C: Chronic 17beta-estradiol treatment improves skeletal muscle insulin signaling pathway components in insulin resistance associated with aging. Age (Dordr) 2010, 32: 1-13.

27. Hamilton RT, Rettberg JR, Mao Z, To J, Zhao L, Appt SE, Register TC,et al.: Hippocampal responsiveness to 17 beta-estradiol and equol after long-term ovariectomy: implication for a therapeutic window of opportunity. Brain Res 2011, 1379: 11-22.

28. Sophonsritsuk A, Appt SE, Clarkson TB, Register TC: Estrogen Therapy and Cardiovascular Disease: Could arterial Expression of Interleukin 17 Provide Some Answers to the Timing Hypothesis? A Study of Early and Late Menopause in Non-Human Primates. Fertil Steril 2010, 94: S43-S44.

29. Chen S, Li J, Zhang Z, Li W, Sun Y, Zhang Q, et al.: Effects of resveratrol on the amelioration of insulin resistance in KKAy mice. Can J Physiol Pharmacol 2012, 90: 237242.

30. Zhu F, Du B, Zheng L, Li J: Advance on the bioactivity and potential applications of dietary fibre from grape pomace. Food Chem 2015, 186: 207-212.

31. Ainslie DA, Morris MJ, Wittert G, Turnbull H, Proietto J, Thorburn AW: Estrogen deficiency causes central leptin insensitivity and increased hypothalamic neuropeptide $\mathrm{Y}$. Int J Obes Relat Metab Disord 2001, 25: 1680-1688.

32. Gavin KM, Cooper EE, Raymer DK, Hickner RC: Estradiol effects on subcutaneous adipose tissue lipolysis in premenopausal women are adipose tissue depot specific and treatment dependent. Am J Physiol Endocrinol Metab 2013, 304: E1167-1174.

33. O'Sullivan AJ, Crampton LJ, Freund J, Ho KK: The route of estrogen replacement therapy confers divergent effects on substrate oxidation and body composition in postmenopausal women. J Clin Invest 1998, 102: 1035-1040. 